\title{
Retrieval-Enhanced Adversarial Training for Neural Response Generation
}

\author{
Qingfu Zhu ${ }^{\sharp}$, Lei Cui ${ }^{b}$, Weinan Zhang ${ }^{\sharp h}$, Furu Wei ${ }^{b}$, Ting Liu ${ }^{\sharp t *}$ \\ ${ }^{\sharp}$ Harbin Institute of Technology, Harbin, China \\ ${ }^{b}$ Microsoft Research Asia, Beijing, China \\ ${ }^{\natural}$ Peng Cheng Laboratory, Shenzhen, China \\ \{qfzhu, wnzhang, tliu\}eir.hit.edu.cn \\ \{lecu, fuwei\}@microsoft.com
}

\begin{abstract}
Dialogue systems are usually built on either generation-based or retrieval-based approaches, yet they do not benefit from the advantages of different models. In this paper, we propose a Retrieval-Enhanced Adversarial Training (REAT) method for neural response generation. Distinct from existing approaches, the REAT method leverages an encoder-decoder framework in terms of an adversarial training paradigm, while taking advantage of $\mathrm{N}$-best response candidates from a retrieval-based system to construct the discriminator. An empirical study on a large scale public available benchmark dataset shows that the REAT method significantly outperforms the vanilla Seq2Seq model as well as the conventional adversarial training approach.
\end{abstract}

\section{Introduction}

Dialogue systems intend to converse with humans with a coherent structure. They have been widely used in real-world applications, including customer service systems, personal assistants, and chatbots. Early dialogue systems are often built using the rule-based method (Weizenbaum, 1966) or template-based method (Litman et al., 2000; Schatzmann et al., 2006; Williams and Young, 2007), which are usually labor-intensive and difficult to scale up. Recently, with the rise of social networking, conversational data have accumulated to a considerable scale. This promoted the development of data-driven methods, including retrieval-based methods (Shang et al., 2015; Sordoni et al., 2015; Vinyals and Le, 2015; Wen et al., 2017) and generation-based methods (Leuski et al., 2006; Ji et al., 2014; Yan et al., 2016).

Retrieval-based methods reply to users by searching and re-ranking response candidates

\footnotetext{
${ }^{*}$ Corresponding author.
}

\begin{tabular}{ll}
\hline MSG & I made strawberry shortcake. \\
\hline GT & Where did you learn that, it is sweet and cheery. \\
RSP & How did you make it? It looks delicious. \\
C\#1 & Could you tell me how this thing is cooked? \\
C\#2 & Tiramisu is my favorite dessert. It's so delicious. \\
\hline
\end{tabular}

Table 1: An example of a message (MSG), a groundtruth response (GT), a generated response (RSP) and $\mathrm{N}$-best response candidates $(\mathrm{C \# 1}$ and $\mathrm{C \# 2}$ ) during the training process. Similar contents in the response and candidates are in boldface.

from a pre-constructed response set. Written mainly by humans, these responses are always diverse and informative, but may be inappropriate to input messages due to their being prepared in advance and thus incapable of being customized (Shang et al., 2015). In contrast, generation-based methods can produce responses tailored to the messages. The most common method of this category in recent years is the sequence to sequence (Seq2Seq) model (Sutskever et al., 2014; Shang et al., 2015; Vinyals and Le, 2015). In practice, it usually suffers from the problem of generating generic responses, such as "I don't know" and "Me, too" (Li et al., 2016a; Serban et al., 2016). While the contents of retrieved responses, apart from the irrelevant parts, are of great diversity, making it a potential resource for tailoring appropriate and informative responses. Therefore, it is natural to enhance the response generation approach with retrieved responses.

Previous work has been proposed to extend the input of a Seq2Seq model with N-best response candidates (or their contexts) (Song et al., 2018; Pandey et al., 2018). On one hand, these approaches are trained using MLE objective, which correlates weakly with true quality of responses thus limits the effectiveness of the candidates in producing the responses. Table 1 shows an exam- 
ple during the training process. Related contents of the candidates are appropriately integrated into the response, but the model is discouraged as the response is different from the ground-truth. On the other hand, rather than just provide materials for the generation, $\mathrm{N}$-best response candidates also contain references for evaluating responses. Yet they are not efficiently utilized in the objective in the existing training process.

In this paper, we propose a Retrieval-Enhanced Adversarial Training (REAT) approach to make better use of N-best response candidates. A discriminator is introduced to replace the MLE objective to supervise the training process. Generated responses containing appropriate and informative contents with input messages are more likely to be seen as human-generated by the discriminator, which encourages the generation model to incorporate more information in candidates into responses. In addition, the N-best response candidates are also conditioned to the discriminator as references to improve its classification accuracy, which in turn benefits the generation model by adversarial training. We conduct extensive experiments on a public available NTCIR corpus to verify the effectiveness of the proposed approach, comparing it with retrievalbased methods, generation-based methods, and previous retrieval-enhanced response generation approaches. The results show that the REAT approach significantly outperforms the baselines in both automatic and human evaluations.

The contributions of this paper are summarized as follows:

1. We propose a novel retrieval-enhanced neural response generation model adapted from adversarial training approach, which introduces a discriminator to more efficiently utilize the $\mathrm{N}$-best response candidates.

2. Referencing to N-best response candidates, the discriminator of our proposed approach improves over previous discriminators on the classification accuracy.

3. Extensive experiments show that our proposed approach outperforms state-of-the-art baselines in both automatic and human evaluations.

\section{Related Work}

Data-driven dialogue systems can be roughly divided into two categories: retrieval-based and generation-based. Retrieval-based methods respond to users by selecting the response that best matches an input message from a pre-constructed response set. Leuski et al. (2006) match a response with a message using a statistical language model. Ji et al.(2014) employ information retrieval techniques to rank response candidates. In addition, the matching and ranking methods can also be implemented using neural networks (Yan et al., 2016; Qiu et al., 2017; Wu et al., 2017). Based on that, Yang et al. (2018) propose a deep matching network which could model external knowledge.

Generation-based methods can be cast as a sequence to sequence (Seq2Seq) process (Shang et al., 2015; Vinyals and Le, 2015; Sordoni et al., 2015) but suffers from generating generic responses. One way to address the problem is to introduce new content into responses, such as keywords (Mou et al., 2016; Serban et al., 2017a), topic information (Xing et al., 2017) and knowledge triples (Zhou et al., 2018). Another way is to improve the Seq2Seq architecture. Li et al.(2016b) introduce the Maximum Mutual Information as the objective function. Serban et al.(2017b) add a latent variable to inject variability. The training of Seq2Seq can be formulated as a reinforcement learning problem ( $\mathrm{Li}$ et al., 2016b; Zhang et al., 2017). To avoid manually defining reward functions, a discriminator can be introduced and trained synchronously by adversarial learning ( $\mathrm{Li}$ et al., 2017). After that, Xu et al. (2018) propose a language model based discriminator to better distinguish novel responses from repeated responses. In a similar adversarial setting, Zhang et al. (2018) optimize a Variational Information Maximization Objective to improve informativeness. Our approach is also an adversarial model, the difference is that we employ the $\mathrm{N}$-best response candidates to enhance the generation.

Taking advantages of the two methods, retrieval-enhanced response generation approaches make use of the informative content in retrieved results to generate new responses. Typically, generating responses from retrieved candidates can be seen as a text-to-text system, which produces meaningful text from meaningful text rather than from abstract meaning representations (Marsi and Krahmer, 2005). Barzilay 


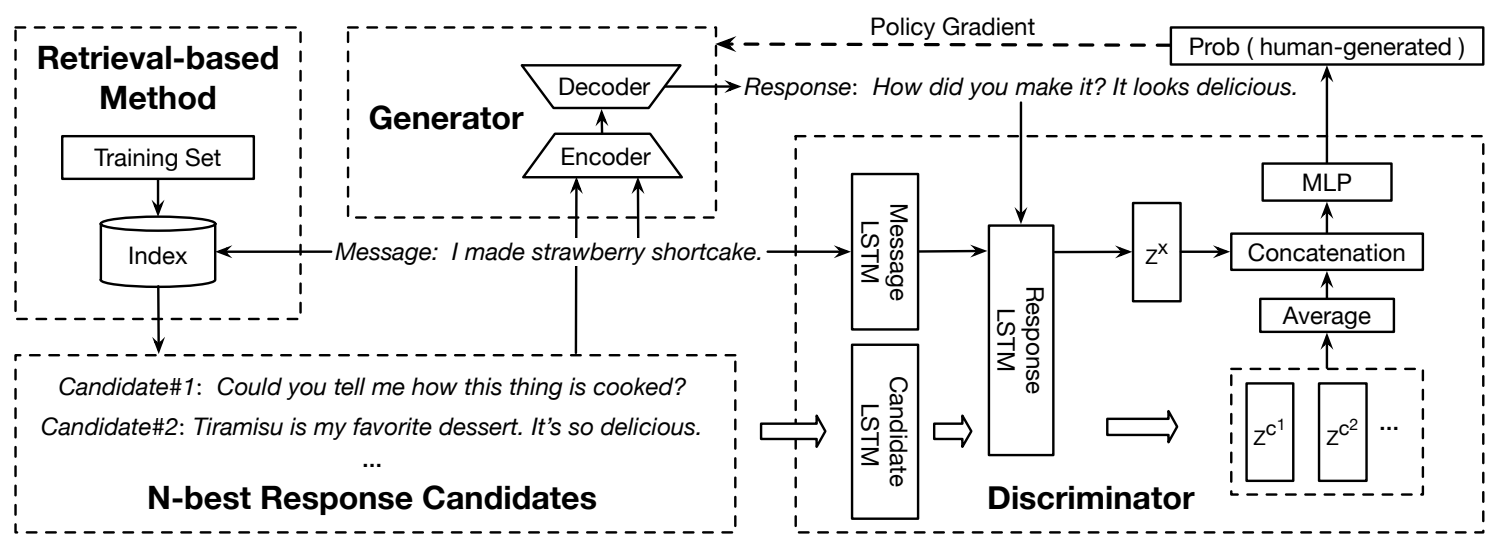

Figure 1: An overview of our proposed approach. The discriminator is enhanced by the N-best response candidates returned by a retrieval-based method. The discriminator takes as input a response and outputs the probability that the response is human-generated. The output is then regarded as a reward to guide the generator.

and McKeown (2005) propose the sentence fusion technique for abstractive multidocument summarization. In the context of conversation, Song et al.(2018) apply an encoder to every response candidate and integrate the results into the decoding process via the attention mechanism (Bahdanau et al., 2015). Similarly, Pandey et al.(2018) also incorporate response candidates using the attentive encoder-decoder framework on a proposed technical support dataset. Wu et al.(2019) augments the decoder with an edit vector representing lexical differences between retrieved contexts and the message. Different from previous work, our approach introduces a discriminator to replace the MLE objective to compute the loss. Besides, rather than merely being sent to the encoder as generation materials, response candidates in our approach are directly utilized by the discriminator to form a discriminative signal to guide the generator. The proposed approach is also related to Lin et al.(2017)'s work. They propose an unconditional GAN whose discriminator is augmented with references randomly sampled from the training set for the task of language generation. In contrast, our proposed approach focuses on the response generation and leverages the message as prior knowledge. In addition, rather than sampling references from the training set, the candidates in our approach are retrieved according to the relevance to messages using a retrieval-based method.

\section{Method}

In this section, we introduce our proposed REAT approach. As Figure 1 shows, it consists of two main components: a discriminator $D$ (Sec. 3.2) and a generator $G$ (Sec. 3.3), both of which are enhanced by N-best response candidates from a retrieval-based method (Sec. 3.4). The generator produces a response using the candidates as generation materials. While in the discriminator, the candidates are provided as references to better distinguish a response, which in turn improves the generator by adversarial training (Sec. 3.1).

\subsection{Retrieval-Enhanced Adversarial Training}

The goal of the discriminator is to distinguish whether a response $y$ is human-generated or machine-generated. It computes the probability $D_{\phi}(y \mid x,\{c\})$ that the response is humangenerated given an input message $x$ and N-best response candidates $\{c\}=\left\{c^{1}, \ldots c^{k}, \ldots, c^{N}\right\}$, where $\phi$ denote the parameters of the discriminator. Therefore, its objective function is to minimize classification error rate:

$$
\begin{aligned}
J_{D}(\phi)= & -\mathbb{E}_{y \sim \text { ground-truth }} \log D_{\phi}(y \mid x,\{c\}) \\
& -\mathbb{E}_{y \sim G} \log \left(1-D_{\phi}(y \mid x,\{c\}),\right.
\end{aligned}
$$

We cast the retrieval-enhanced response generation as a reinforcement learning problem to backpropagate the error computed by the discriminator to the generator via the policy gradient algorithm. In this way, the generator can be seen as an agent whose parameters $\theta$ define a policy $\pi$. At each time step, it takes an action $a$ by generating a word and accordingly updates its state $s$, which is defined as a tuple of the message, the candidates and the partially generated response. At the end of the generation of a response, the agent observes a reward $r$ from the discriminator, which is the probability that the response is 
human-generated: $D_{\phi}(y \mid x,\{c\})$. Here, we do not employ the REGS (reward for every generation step) strategy (Li et al., 2017) as the Monte-Carlo roll-out is quite time-consuming ${ }^{1}$ and the accuracy of a discriminator trained on partially decoded sequences is not as good as that trained on complete sequences.

The goal of the agent (the generator) is to minimize the negative expected reward. With the likelihood ratio trick (Williams, 1992), the gradient of $\theta$ can be derived as:

$$
\begin{aligned}
J_{G}(\theta)= & -\mathbb{E}_{y \sim G}\left(D_{\phi}(y \mid x,\{c\})\right), \\
\nabla J_{G}(\theta)= & -\mathbb{E}_{y \sim G}\left(D_{\phi}(y \mid x,\{c\})\right. \\
& \left.\cdot \nabla \log G_{\theta}(y \mid x,\{c\})\right),
\end{aligned}
$$

where $G_{\theta}(y \mid x,\{c\})$ is the probability of generating $y$ given $x$ and $\{c\}$. In practice, $J_{G}(\theta)$ and $\nabla J_{G}(\theta)$ can be approximated using a single Monte-Carlo sample from $G$ (Rennie et al., 2017):

$$
\begin{aligned}
J_{G}(\theta) \approx & -D_{\phi}(y \mid x,\{c\}), \quad y \sim G, \\
\nabla J_{G}(\theta) \approx & -D_{\phi}(y \mid x,\{c\}) \\
& \cdot \nabla \log G_{\theta}(y \mid x,\{c\}), \quad y \sim G .
\end{aligned}
$$

Both the generator and the discriminator are pre-trained before adversarial training. The generator is pre-trained on the training set with MLE loss. The discriminator is pre-trained using human-generated responses as positive samples and machine-generated responses produced by the pre-trained generator as negative samples.

Given the pre-trained generator and discriminator, the adversarial training is a min-max game played between them:

$$
\min _{G} \max _{D} J_{G}(\theta)-J_{D}(\phi),
$$

where the discriminator tries to distinguish between human-generated responses and machinegenerated responses, while the generator tries to fool the discriminator by producing human-like responses. The overall algorithm of the retrievalenhanced adversarial training is summarized as Algorithm 1.

\subsection{Discriminator}

The discriminator is a binary classifier. It takes as input a response $y$, a message $x$, and N-best

\footnotetext{
${ }^{1}$ Training one epoch takes roughly 120 hours on a TITAN $\mathrm{Xp} \mathrm{GPU}$ when the roll-out number is 5.
}

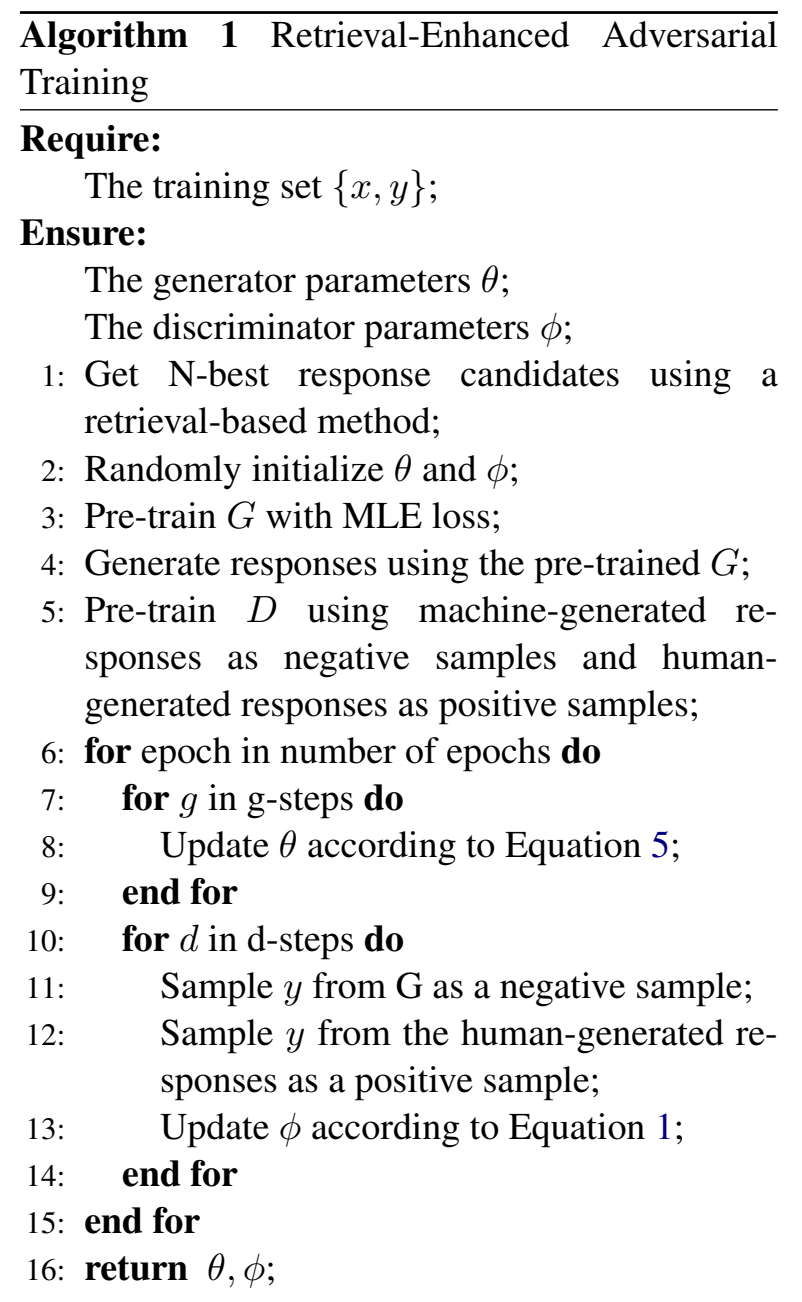

response candidates $\{c\}$, and subsequently computes a binary probability distribution to indicate whether $y$ is human-generated or machinegenerated.

First, we compute a candidate-aware response representation $z^{c}$ to model the interaction between the candidates and the response. Each candidate is encoded by a candidate LSTM (Hochreiter and Schmidhuber, 1997):

$$
u_{i}^{k}=f_{c}\left(c_{i}^{k}, u_{i-1}^{k}\right)
$$

where $c_{i}^{k}$ is the $i$-th word of the $k$-th candidate. $u_{i}^{k}$ denotes the hidden state of the candidate LSTM at time step $i . f_{c}$ is the computation unit of the candidate LSTM. The initial hidden state $u_{0}^{k}$ is set to the zero vector and the last hidden state $u_{T}^{k}$ (T denotes the length of an utterence through out the paper) can be seen as a representation of the candidate. Subsequently, $u_{T}^{k}$ is used to initialize the hidden state of a response LSTM, which computes a local candidate-aware response representation $z^{c^{k}}$ 
for each candidate $c^{k}$ :

$$
v_{i}^{k}=f_{y}\left(y_{i}, v_{i-1}^{k}\right), \quad z^{c^{k}}=v_{T}^{k},
$$

where $v_{i}^{k}$ represents the hidden state of the response LSTM at time step $i$ with regard to the $k$-th candidate. $f_{y}$ is the computation unit of the response LSTM and $y_{i}$ is the $i$-th word of the response. The candidate-aware response representation $z^{c}$ is the average of all local candidate-aware response representations:

$$
z^{c}=\frac{1}{N} \sum_{k=1}^{N} z^{c^{k}},
$$

Second, the interaction between the message and the response is modeled by a message-aware response representation $z^{x}$ using a message LSTM and the response LSTM introduced above in a similar way to Equation 7 and 8.

Finally, the probability that the response is human-generated $D_{\phi}(y \mid x,\{c\})$ is computed by a Multilayer Perception (MLP):

$$
D_{\phi}(y \mid x,\{c\})=\sigma\left(\operatorname{MLP}\left(\left[z^{x}, z^{c}\right]\right)\right),
$$

where the bracket $[\cdot, \cdot]$ denotes concatenation. $\sigma$ is the sigmoid function ${ }^{2}$.

\subsection{Generator}

The generator $G$ is a multi-source Seq2Seq model, which consists of an encoder and a decoder. The encoder reads from a message and $\mathrm{N}$-best response candidates, summarizing them into context vectors. The decoder is a language model which produces a response word by word, conditioned with the context vectors.

The encoder first employs a bi-directional LSTM to represent each candidate word and its context information in a response candidate:

$$
\overrightarrow{h_{i}^{k}}=g_{c}^{0}\left(c_{i}^{k}, \overrightarrow{h_{i-1}^{k}}\right), \quad \overleftarrow{h_{i}^{k}}=g_{c}^{1}\left(c_{i}^{k}, h_{i+1}^{\overleftarrow{k}}\right)
$$

where $g_{c}^{0}$ and $g_{c}^{1}$ denote the computation units of a forward LSTM and a backward LSTM, respectively. $\overrightarrow{h_{i}^{k}}$ and $\overleftarrow{h_{i}^{k}}$ are the $i$-th hidden states of the two LSTMs. After that, hidden states in the two directions are concatenated, i.e., $h_{i}^{k}=\left[\overrightarrow{h_{i}^{k}}, \overleftarrow{h_{i}^{k}}\right]$.

\footnotetext{
${ }^{2}$ We did study more complicated relationship among $x, y$ ,and $\{c\}$ with bi-directional LSTM and attention mechanism in the discriminator, but observed no further improvement on the validation set.
}

To capture the different importance of a candidate word in the word-level and the sentence-level, the encoder employs a two-level attention structure. The word-level attention models the relevance of a candidate word to the decoding context within a candidate, i.e, the word-level attention at the $j$-th decoding time step is computed as:

$$
\alpha_{i j}^{k}=\frac{\exp \left(q\left(s_{j-1}, h_{i}^{k}\right)\right)}{\sum_{t=1}^{T} \exp \left(q\left(s_{j-1}, h_{t}^{k}\right)\right)},
$$

where $\alpha_{i j}^{k}$ is the word-level weight for the $i$-th word of $c^{k} . s_{j-1}$ is the hidden state of the decoder, representing the decoding context at time step $j . q$ is a feed-forward network. Considering that different candidates are of different importance, the word-level weights are then rescaled by a sentence-level attention:

$$
\begin{gathered}
a_{j}^{c^{k}}=\sum_{i=1}^{T} \alpha_{i j}^{k} h_{i}^{k}, \\
\beta_{k j}=\frac{\exp \left(q\left(s_{j-1}, a_{j}^{c^{k}}\right)\right)}{\sum_{n=1}^{N} \exp \left(q\left(s_{j-1}, a_{j}^{c^{n}}\right)\right)} .
\end{gathered}
$$

where $a_{j}^{c^{k}}$ can be seen as a representation of $c^{k}$. $\beta_{k j}$ is the sentence-level weight of $c^{k}$. The candidate context vector $a_{j}^{c}$ is then computed taking into account the two-level attention weights:

$$
a_{j}^{c}=\sum_{k=1}^{N} \sum_{i=1}^{T} \beta_{k j} \alpha_{i j}^{k} h_{i}^{k}
$$

Meanwhile, the message context vector $a_{j}^{x}$ is computed using a message bi-directional LSTM and a word-level attention in a similar way to Equation 11, 12 and 13. Then, the decoder LSTM updates its hidden state conditioned with the context vectors and subsequently generates a word for a response as a standard language model:

$$
s_{j}=g_{y}\left(\left[y_{j-1}, a_{j}^{c}, a_{j}^{x}\right], s_{j-1}\right) .
$$

where $g_{y}$ is the computation unit of the decoder.

\subsection{Retrieval-based Method}

To get the N-best response candidates, a retrievalbased method is built using the Lucene ${ }^{3}$ library and the state-of-the-art response ranking model (Yang et al., 2018). First, we merge all

\footnotetext{
${ }^{3}$ https://lucene.apache.org/
} 


\begin{tabular}{lrr}
\hline Corpus & \# of message & \# of response \\
\hline Training & 119,941 & $1,932,258$ \\
Validation & 10,000 & 163,126 \\
Test & 10,000 & 162,230 \\
\hline
\end{tabular}

Table 2: Some statistics of the datasets.

message-response pairs whose messages are identical into a document and subsequently build the index for all the documents in the training set. Second, we use each message as a query to search for $K$ (set to 10) documents whose messages are similar to the query. After that, responses in the retrieved documents are re-ranked by the ranking model according to their matching scores to the query. Finally, the top $N$ (set to 2, as in Song et al., 2018) responses are returned as the N-best response candidates.

Note that when we collect N-best response candidates for a training message, the most similar document retrieved is always the one whose message is exactly the training message and responses contain the ground-truth response. We thus remove the document from the retrieved result before re-ranking to make sure that the N-best response candidates are different from the groundtruth response.

\section{Experiments}

\subsection{Data}

We use the NTCIR corpus ${ }^{4}$ in our experiments. Its data are collected from a Chinese microblogging service, Sina Weibo ${ }^{5}$, where users can both post messages and make comments (responses) on other users' messages. First, we tokenize each utterance using the Language Technology Platform (Che et al., 2010) and remove samples whose responses are shorter than 5 , which is helpful in relieving the generic response problem ( $\mathrm{Li}$ et al., 2017). Then, we randomly select 10,000 messages associated with responses to form a validation set and another 10,000 messages with responses as a test set. Table 2 shows some statistics of the datasets.

\subsection{Baselines}

Rtr: The retrieval-based method searches the index for response candidates and subsequently re-

\footnotetext{
${ }^{4}$ http://research.nii.ac.jp/ntcir/data/data-en.html

${ }^{5}$ https://weibo.com
}

turns the one that best matches the message after re-ranking (see Sec. 3.4 for details).

S2S: The Seq2Seq model with the attention mechanism (Bahdanau et al., 2015).

MS2S: The "multi sequence to sequence" (Song et al., 2018) encodes N-best response candidates using $N$ encoders and subsequently incorporates the results into the decoding process by the attention mechanism.

Edit: The prototype editing model (Wu et al., 2019) augments the decoder with an edit vector representing lexical differences between retrieved contexts and the message.

AL: The adversarial learning for neural response generation (Li et al., 2017) is also an adversarial method but is not retrieval-enhanced. Here, we do not employ the REGS (reward for every generation step) setting as the Monte-Carlo roll-out is quite time-consuming and the accuracy of the discriminator trained on partially decoded sequences is not as good as that on complete sequences.

\subsection{Experiment Settings}

We use the published code ${ }^{6}$ for Edit and implement other approaches by an open source framework: Open-NMT (Klein et al., 2017). The vocabulary table consists of the most frequent 30,000 words, whose 300-dimensional word embeddings are pre-trained on the training set by Word2 Vec ${ }^{7}$. The number of hidden units for all LSTM in our approach is 500. The batch size is set to 64 .

The discriminator and the generator are trained alternately, where the discriminator is optimized for 10 batches, then switch to the generator for 20 batches. We use ADAM optimizer whose learning rate is initialized to 0.0001 . In the inference process, we generate responses using beam search with beam size set to 5 .

\section{Results}

\subsection{Evaluation Metrics}

Human Evaluation We randomly sampled 200 messages from the test set to conduct the human evaluation as it is extremely time-consuming. Five annotators ${ }^{8}$ are recruited to judge a response from three aspects (Ke et al., 2018):

\footnotetext{
${ }^{6}$ https://github.com/MarkWuNLP/ResponseEdit

${ }^{7}$ https://code.google.com/archive/p/word2vec/

${ }^{8}$ All annotators are well-educated students and have Bachelor or higher degree.
} 


\begin{tabular}{l|rrrr|rrrrrrr|rrrrrr}
\hline & \multicolumn{7}{|c|}{ Appropriateness } & \multicolumn{5}{c}{ Informativeness } & \multicolumn{5}{c}{ Grammaticality } \\
& Mean & +2 & +1 & 0 & $\kappa$ & Mean & +2 & +1 & 0 & $\kappa$ & Mean & +2 & +1 & 0 & $\kappa$ \\
\hline Rtr & 0.63 & 24.8 & 12.9 & 62.3 & 0.71 & 0.92 & 41.1 & 10.1 & 48.8 & 0.67 & 1.93 & 94.9 & 3.1 & 2.0 & 0.61 \\
S2S & 0.76 & 27.9 & 20.0 & 52.1 & 0.58 & 0.51 & 10.2 & 30.5 & 59.3 & 0.69 & 1.74 & 85.3 & 2.9 & 11.8 & 0.83 \\
MS2S & 0.85 & 31.9 & 21.5 & 46.6 & 0.63 & 0.62 & 14.1 & 33.8 & 52.1 & 0.73 & 1.74 & 85.5 & 3.2 & 11.3 & 0.82 \\
Edit & 0.85 & 31.4 & 21.9 & 46.7 & 0.66 & 0.67 & 15.9 & 34.9 & 49.2 & 0.68 & 1.92 & 95.2 & 1.5 & 3.3 & 0.63 \\
AL & 0.98 & 36.8 & 24.0 & 39.2 & 0.57 & 0.77 & 21.8 & 33.6 & 44.6 & 0.66 & 1.88 & 91.7 & 4.7 & 3.6 & 0.58 \\
Ours & 1.10 & 41.5 & 26.8 & 31.7 & 0.65 & 0.88 & 31.2 & 25.9 & 42.9 & 0.72 & 1.87 & 89.6 & 7.6 & 2.8 & 0.60 \\
\hline
\end{tabular}

Table 3: Human evaluation results of mean score, proportions of three levels $(+2,+1$, and 0$)$, and the agreements measured by Fleiss's Kappa in appropriateness, informativeness, and grammaticality.

\begin{tabular}{ccc}
\hline & AL & Ours \\
\hline Accuracy & $94.01 \%$ & $95.72 \%$ \\
\hline
\end{tabular}

Table 4: Classification accuracy of discriminators in AL and our approach.

- appropriateness: a response is logical and appropriate to its message.

- informativeness: a response has meaningful information relevant to its message.

- grammaticality: a response is fluent and grammatical.

These aspects are evaluated independently. For each aspect, three levels are assigned to a response with scores from 0 to +2 (Shang et al., 2015), where 0 represents bad and +2 represents excellent. The appropriateness differs from the informativeness in that the former focuses on the logical relationship between a message and a response, while the latter evaluates the richness of relevant content.

Automatic Evaluation We employ Dist-1 and Dist-2 (Li et al., 2016a) to evaluate the diversity of responses, where Dist-k is the number of distinct $k$-grams normalized by the total number of words of responses. We also evaluate the Originality by computing the ratio of responses that do not appear in the training set (Wu et al., 2019).

To validate the effectiveness of retrieved candidates in enhancing the discriminator, the classification accuracy of the discriminator in AL and our approach is also reported. Note that the two discriminators after pre-training or adversarial training cannot be compared directly because they are trained by different negative samples produced by different generators. We thus create a special dataset for this metric where negative samples are generated by a well-trained generator (otherwise, the accuracy will easily reach nearly $100 \%$ as fixed negative samples of low quality are too easy to be distinguished) of AL in advance.

\subsection{Analysis}

The results of the classification accuracy of different discriminators are presented in Table 4. Trained on an identical dataset, our discriminator achieves higher accuracy than the conventional discriminator in AL. This indicates that the N-best response candidates are helpful for the discriminator in distinguishing between human-generated responses and machine-generated responses, which could in turn benefit the generator in the adversarial training process (discussed later).

Table 3 shows the results of human evaluation. Our approach has the highest mean score and the largest proportions of +2 and +1 in appropriateness. Meanwhile, it outperforms all generationbased and retrieval-enhanced approaches in informativeness. This suggests that our approach is able to respond more appropriately and incorporate informative content into responses at the same time. Note that Rtr has the highest informativeness mean score due to its diverse human-written content. However, it may also contain some irrelevant information, leading to a bad performance in appropriateness. Besides, most responses in Rtr are annotated as +2 or 0 in informativeness. This is also because Rtr responses are extremely diverse which always include new content, making a response tend to get +2 if the content is relevant, otherwise 0 . In terms of grammaticality, the mean score of our approach is higher than that of S2S and MS2S, and is comparable with that of $\mathrm{AL}$, indicating that our approach is competitive in generating fluent responses. Edit has a high mean score mainly due to its relatively simple sentence structure. As shown in Figure 2, S2S and MS2S have similar simple sentence structure to 


\begin{tabular}{lrrrrr}
\hline Model & \# of UNI & Dist-1 & \# of BI & Dist-2 & Origin \\
\hline Rtr & 6,837 & 0.113 & 25,863 & 0.428 & 0.000 \\
S2S & 1,247 & 0.023 & 3,122 & 0.060 & 0.288 \\
MS2S & 2,596 & 0.049 & 6,455 & 0.122 & 0.351 \\
EDIT & 1,847 & 0.027 & 5,690 & 0.085 & 0.540 \\
AL & 1,760 & 0.033 & 6,697 & 0.124 & 0.590 \\
\hline D+ & 2,057 & 0.038 & 8,683 & 0.158 & 0.775 \\
G+ & 2,440 & 0.046 & 10,461 & 0.200 & 0.792 \\
Ours & 3,356 & 0.060 & 13,184 & 0.236 & 0.842 \\
\hline
\end{tabular}

Table 5: Automatic evaluation results of the number of distinct uni-grams (\# of UNI) and bi-grams (\# of BI), Dist-1, Dist-2 and Originality (Origin). D+ and $\mathrm{G}+$ are two variants of our approach where candidates are only available for the discriminator and the generator, respectively.

Edit, the reason for the relatively low mean scores of $\mathrm{S} 2 \mathrm{~S}$ and MS2S in grammaticality is that they have some repetitive responses, like "windows, windows, windows".

Agreements among different annotators are calculated by Fleiss' Kappa (Fleiss, 1971). The values of appropriateness and informativeness are all in an interval of $(0.4,0.6]$ or $(0.6,0.8]$, which can be seen as "Moderate agreement" and "Substantial agreement", respectively. Grammaticality has relatively higher agreement values as it is easier to reach an agreement on grammatical errors.

We report the results of Dist-1, Dist-2, and Originality in Table 5. AL outperforms S2S in all metrics, indicating that adversarial training is helpful for generating diverse n-grams and responses. By introducing N-best response candidates, our approach further increases Dist-2 by 0.112 based on AL (from 0.124 to 0.236 ) and the improvement is significant (t-test, $p<0.01$ ). In contrast, the increase of Dist- 2 after combining Nbest response candidates in MLE based approach is only 0.062, comparing MS2S with S2S. This suggests that introducing a discriminator with adversarial training is more effective than MLE objective in utilizing $\mathrm{N}$-best response candidates to generate more diverse n-grams. Note that the improvement after introducing candidates in Dist-1 and Originality is not as significant as that in Dist2. This is because responses of MLE based models (MS2S and EDIT) tend to contain informative content with simple sentence structures, like "... is (not) good." (as shown in Figure 2), resulting in high Dist- 1 and Originality scores, but their Dist-2 scores are relatively lower than AL and Ours.

To understand the importance of different com-

\begin{tabular}{|c|c|c|}
\hline & Utterance & Translation \\
\hline MSG & $\begin{array}{l}\text { 家里有Wi-Fi信号 } \\
\text { 但连不上网啊, 什 } \\
\text { 么原因啊? }\end{array}$ & $\begin{array}{r}\text { I have a Wi-Fi signal at } \\
\text { home, but do not have } \\
\text { access to the Internet, } \\
\text { what's the reason? }\end{array}$ \\
\hline $\mathrm{C} \# 1$ & $\begin{array}{l}\text { 我猜是电信跟联通 } \\
\text { 通话时有问题。 }\end{array}$ & $\begin{array}{l}\text { I guess there is a problem } \\
\text { with the call between } \\
\text { Telecom and Unicom. }\end{array}$ \\
\hline $\mathrm{C} \# 2$ & $\begin{array}{l}\text { 我觉得不是你的问 } \\
\text { 题。 }\end{array}$ & $\begin{array}{l}\text { I don't think it's your } \\
\text { problem. }\end{array}$ \\
\hline $\mathrm{S} 2 \mathrm{~S}$ & 我也是这么想的。 & I think so too. \\
\hline MS2S & 我的手机信号不好。 & $\begin{array}{r}\text { My cell phone signal is } \\
\text { not good. }\end{array}$ \\
\hline EDIT & 这个广告太电信了。 & This ad is too Telecom. \\
\hline $\mathrm{AL}$ & 没信号就没信号了。 & No signal, no signal. \\
\hline Ours & 换成联通手机吧。 & $\begin{array}{l}\text { Let's change to } \\
\text { Unicom's mobile phone. }\end{array}$ \\
\hline
\end{tabular}

Figure 2: An example of a test message (MSG), candidates (C\#1 and $\mathrm{C \# 2}$ ), and responses from different models. The last column are their translations.

ponents of our approach, we also train two variants: D+ and G+, where N-best response candidates are only available for the discriminator and the generator, respectively. Note that AL does not utilize candidates in the generator nor the discriminator, thus can be seen as a start point of D+ and G+. As shown in Table 5, there is an improvement in the performance of both the two variants after introducing the candidates comparing to AL. The improvement in $\mathrm{G}+$ is more significant as its generator can directly utilize the candidates as generation materials. While candidates' information in $\mathrm{D}+$ is compressed into a discriminative signal by the discriminator. Nevertheless, introducing candidates into the discriminator helps to generate more diverse responses comparing $\mathrm{AL}$ with $\mathrm{D}+$, and $\mathrm{G}+$ with Ours, demonstrating that the retrieval-enhanced discriminator is able to benefit the generator.

Figure 2 shows an example of responses of different models along with the input message and $\mathrm{N}$ -

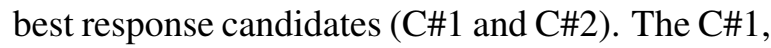
which best matches the message among all the candidates, is also the response of the Rtr baseline. We can see that it contains diverse content, such as "Unicom" and "Telecom"(two telecommunication operators in China, providing broadband, mobile communication as well as customized mobile phones). However, it talks about "the call" be- 
tween the two operators, which is irrelevant to the message. The response of $\mathrm{S} 2 \mathrm{~S}$ is a generic response. AL has a more diverse response than S2S, however, it does not have access to candidates, which limits the diversity. MLE based retrievalenhanced models can make use of the content of candidates, like "Telecom" in EDIT, but the way they present the content is not as diverse as ours.

\section{Conclusion and Future Work}

We propose a Retrieval-Enhanced Adversarial Training method for neural response generation in dialogue systems. In contrast to existing approaches, our REAT method directly uses response candidates from retrieval-based systems to improve the discriminator in adversarial training. Therefore, it can benefit from the advantages of retrieval-based response candidates as well as neural responses from generation-based systems. Experiments show that the REAT method significantly improves the quality of the generated responses, which demonstrates the effectiveness of this approach.

In future research, we will further investigate how to better leverage larger training data to improve the REAT method. In addition, we will also explore how to integrate external knowledge in other formats, like the knowledge graph, into adversarial training so that the quality could be further improved.

\section{Acknowledgments}

The authors would like to thank all the anonymous reviewer for their insightful comments. The paper is supported by the National Natural Science Foundation of China (No. 61772153).

\section{References}

Dzmitry Bahdanau, Kyunghyun Cho, and Yoshua Bengio. 2015. Neural machine translation by jointly learning to align and translate. In Proceedings of International Conference on Learning Representations.

Regina Barzilay and Kathleen R McKeown. 2005. Sentence fusion for multidocument news summarization. Journal of Computational Linguistics, 31(3):297-328.

Wanxiang Che, Zhenghua Li, and Ting Liu. 2010. LTP: A Chinese language technology platform. In Coling 2010: Demonstrations, pages 13-16, Beijing, China. Coling 2010 Organizing Committee.
Joseph L Fleiss. 1971. Measuring nominal scale agreement among many raters. Journal of Psychological bulletin, 76(5):378.

Sepp Hochreiter and Jürgen Schmidhuber. 1997. Long short-term memory. Journal of Neural computation, 9(8):1735-1780.

Zongcheng Ji, Zhengdong Lu, and Hang Li. 2014. An information retrieval approach to short text conversation. arXiv preprint arXiv:1408.6988.

Pei Ke, Jian Guan, Minlie Huang, and Xiaoyan Zhu. 2018. Generating informative responses with controlled sentence function. In Proceedings of the 56th Annual Meeting of the Association for Computational Linguistics (Volume 1: Long Papers), pages 1499-1508, Melbourne, Australia. Association for Computational Linguistics.

Guillaume Klein, Yoon Kim, Yuntian Deng, Jean Senellart, and Alexander Rush. 2017. OpenNMT: Open-source toolkit for neural machine translation. In Proceedings of ACL 2017, System Demonstrations, pages 67-72, Vancouver, Canada. Association for Computational Linguistics.

Anton Leuski, Ronakkumar Patel, David Traum, and Brandon Kennedy. 2006. Building effective question answering characters. In Proceedings of the 7th SIGdial Workshop on Discourse and Dialogue, pages 18-27, Sydney, Australia. Association for Computational Linguistics.

Jiwei Li, Michel Galley, Chris Brockett, Jianfeng Gao, and Bill Dolan. 2016a. A diversity-promoting objective function for neural conversation models. In Proceedings of the 2016 Conference of the North American Chapter of the Association for Computational Linguistics: Human Language Technologies, pages 110-119, San Diego, California. Association for Computational Linguistics.

Jiwei Li, Will Monroe, Alan Ritter, Dan Jurafsky, Michel Galley, and Jianfeng Gao. 2016b. Deep reinforcement learning for dialogue generation. In Proceedings of the 2016 Conference on Empirical Methods in Natural Language Processing, pages 11921202, Austin, Texas. Association for Computational Linguistics.

Jiwei Li, Will Monroe, Tianlin Shi, Sébastien Jean, Alan Ritter, and Dan Jurafsky. 2017. Adversarial learning for neural dialogue generation. In Proceedings of the 2017 Conference on Empirical Methods in Natural Language Processing, pages 2157-2169, Copenhagen, Denmark. Association for Computational Linguistics.

Kevin Lin, Dianqi Li, Xiaodong He, Zhengyou Zhang, and Ming-Ting Sun. 2017. Adversarial ranking for language generation. In Proceedings of the ThirtyFirst Conference on Neural Information Processing Systems, pages 3155-3165. 
Diane Litman, Satinder Singh, Michael Kearns, and Marilyn Walker. 2000. Njfun: a reinforcement learning spoken dialogue system. In $A N L P-N A A C L$ 2000 Workshop: Conversational Systems, pages 1720.

Erwin Marsi and Emiel Krahmer. 2005. Explorations in sentence fusion. In Proceedings of the Tenth European Workshop on Natural Language Generation (ENLG-05).

Lili Mou, Yiping Song, Rui Yan, Ge Li, Lu Zhang, and Zhi Jin. 2016. Sequence to backward and forward sequences: A content-introducing approach to generative short-text conversation. In Proceedings of COLING 2016, the 26th International Conference on Computational Linguistics: Technical Papers, pages 3349-3358, Osaka, Japan. The COLING 2016 Organizing Committee.

Gaurav Pandey, Danish Contractor, Vineet Kumar, and Sachindra Joshi. 2018. Exemplar encoder-decoder for neural conversation generation. In Proceedings of the 56th Annual Meeting of the Association for Computational Linguistics (Volume 1: Long Papers), pages 1329-1338, Melbourne, Australia. Association for Computational Linguistics.

Minghui Qiu, Feng-Lin Li, Siyu Wang, Xing Gao, Yan Chen, Weipeng Zhao, Haiqing Chen, Jun Huang, and Wei Chu. 2017. AliMe chat: A sequence to sequence and rerank based chatbot engine. In Proceedings of the 55th Annual Meeting of the Association for Computational Linguistics (Volume 2: Short Papers), pages 498-503, Vancouver, Canada. Association for Computational Linguistics.

Steven J Rennie, Etienne Marcheret, Youssef Mroueh, Jerret Ross, and Vaibhava Goel. 2017. Self-critical sequence training for image captioning. In Proceedings of the IEEE Conference on Computer Vision and Pattern Recognition, pages 7008-7024.

Jost Schatzmann, Karl Weilhammer, Matt Stuttle, and Steve Young. 2006. A survey of statistical user simulation techniques for reinforcement-learning of dialogue management strategies. Journal of The Knowledge Engineering Review, 21(2):97-126.

Iulian V Serban, Alessandro Sordoni, Yoshua Bengio, Aaron Courville, and Joelle Pineau. 2016. Building end-to-end dialogue systems using generative hierarchical neural network models. In Proceedings of the Thirtieth AAAI Conference on Artificial Intelligence.

Iulian Vlad Serban, Tim Klinger, Gerald Tesauro, Kartik Talamadupula, Bowen Zhou, Yoshua Bengio, and Aaron C Courville. 2017a. Multiresolution recurrent neural networks: An application to dialogue response generation. In Proceedings of the Thirty-First AAAI Conference on Artificial Intelligence, pages 3288-3294.
Iulian Vlad Serban, Alessandro Sordoni, Ryan Lowe, Laurent Charlin, Joelle Pineau, Aaron Courville, and Yoshua Bengio. 2017b. A hierarchical latent variable encoder-decoder model for generating dialogues. In Proceedings of the Thirty-First AAAI Conference on Artificial Intelligence.

Lifeng Shang, Zhengdong Lu, and Hang Li. 2015. Neural responding machine for short-text conversation. In Proceedings of the 53rd Annual Meeting of the Association for Computational Linguistics and the 7th International Joint Conference on Natural Language Processing (Volume 1: Long Papers), pages 1577-1586, Beijing, China. Association for Computational Linguistics.

Yiping Song, Rui Yan, Cheng-Te Li, Jian-Yun Nie, Ming Zhang, and Dongyan Zhao. 2018. An ensemble of retrieval-based and generation-based humancomputer conversation systems. In Proceedings of the 27th International Joint Conference on Artificial Intelligence and the 23rd European Conference on Artificial Intelligence.

Alessandro Sordoni, Michel Galley, Michael Auli, Chris Brockett, Yangfeng Ji, Margaret Mitchell, Jian-Yun Nie, Jianfeng Gao, and Bill Dolan. 2015. A neural network approach to context-sensitive generation of conversational responses. In Proceedings of the 2015 Conference of the North American Chapter of the Association for Computational Linguistics: Human Language Technologies, pages 196-205, Denver, Colorado. Association for Computational Linguistics.

Ilya Sutskever, Oriol Vinyals, and Quoc V Le. 2014. Sequence to sequence learning with neural networks. In Proceedings of the Twenty-Eighth Conference on Neural Information Processing Systems, pages 3104-3112.

Oriol Vinyals and Quoc Le. 2015. A neural conversational model. arXiv preprint arXiv:1506.05869.

Joseph Weizenbaum. 1966. Eliza-a computer program for the study of natural language communication between man and machine. Journal of Communications of the ACM, 9(1):36-45.

Tsung-Hsien Wen, David Vandyke, Nikola Mrkšić, Milica Gasic, Lina M. Rojas Barahona, Pei-Hao Su, Stefan Ultes, and Steve Young. 2017. A networkbased end-to-end trainable task-oriented dialogue system. In Proceedings of the 15th Conference of the European Chapter of the Association for Computational Linguistics: Volume 1, Long Papers, pages 438-449, Valencia, Spain. Association for Computational Linguistics.

Jason D Williams and Steve Young. 2007. Partially observable markov decision processes for spoken dia$\log$ systems. Journal of Computer Speech \& Language, 21(2):393-422. 
Ronald J Williams. 1992. Simple statistical gradientfollowing algorithms for connectionist reinforcement learning. Journal of Machine Learning, 3(8):229-256.

Yu Wu, Furu Wei, Shaohan Huang, Zhoujun Li, and Ming Zhou. 2019. Response generation by contextaware prototype editing. In Proceedings of the Thirty-Third AAAI Conference on Artificial Intelligence.

$\mathrm{Yu} \mathrm{Wu}$, Wei Wu, Chen Xing, Can Xu, Zhoujun Li, and Ming Zhou. 2017. A sequential matching framework for multi-turn response selection in retrievalbased chatbots. arXiv preprint arXiv:1710.11344.

Chen Xing, Wei Wu, Yu Wu, Jie Liu, Yalou Huang, Ming Zhou, and Wei-Ying Ma. 2017. Topic aware neural response generation. In Proceedings of the Thirty-First AAAI Conference on Artificial Intelligence, volume 17, pages 3351-3357.

Jingjing Xu, Xuancheng Ren, Junyang Lin, and Xu Sun. 2018. Diversity-promoting GAN: A crossentropy based generative adversarial network for diversified text generation. In Proceedings of the 2018 Conference on Empirical Methods in Natural Language Processing, pages 3940-3949, Brussels, Belgium. Association for Computational Linguistics.

Rui Yan, Yiping Song, and Hua Wu. 2016. Learning to respond with deep neural networks for retrievalbased human-computer conversation system. In Proceedings of the 39th International ACM SIGIR Conference on Research and Development in Information Retrieval, pages 55-64. ACM.

Liu Yang, Minghui Qiu, Chen Qu, Jiafeng Guo, Yongfeng Zhang, W Bruce Croft, Jun Huang, and Haiqing Chen. 2018. Response ranking with deep matching networks and external knowledge in information-seeking conversation systems. In Proceedings of The 41 st International ACM SIGIR Conference on Research and Development in Information Retrieval, pages 245-254.

Weinan Zhang, Lingzhi Li, Dongyan Cao, and Ting Liu. 2017. Exploring implicit feedback for open domain conversation generation. In Proceedings of the Thirty-Second AAAI Conference on Artificial Intelligence, pages 547-554.

Yizhe Zhang, Michel Galley, Jianfeng Gao, Zhe Gan, and Bill Dolan. 2018. Generating informative and diverse conversational responses via adversarial information maximization. In Proceedings of the Thirty-Second Conference on Neural Information Processing Systems, pages 1810-1820.

Hao Zhou, Tom Young, Minlie Huang, Haizhou Zhao, Jingfang $\mathrm{Xu}$, and Xiaoyan Zhu. 2018. Commonsense knowledge aware conversation generation with graph attention. In the 27th International Joint Conference on Artificial Intelligence and the 23rd European Conference on Artificial Intelligence, pages 4623-4629. 